\title{
Análise da alteração sensorial em alimentos preparados com suplementos em pó sem sabor
}

\author{
Analysis of sensory changes in foods prepared with tasteless powder supplements
}

\section{DOI: $10.37111 /$ braspenj.2020351007}

Lidiane Pereira Magalhães Júlia de Castro Querido² Mayara Freitas de Oliveira ${ }^{3}$ Nathalia Soares Machado 4 Luciana Pellegrini Pisani ${ }^{5}$

\section{Unitermos:}

Nutrição em Saúde Pública. Oncologia. Suplementos nutricionais. Desnutrição.

\section{Keywords:}

Nutrition, Public Health. Medical oncology. Dietary supplements. Malnutrition.

\section{Endereço para correspondência:}

Lidiane Pereira Magalhães

Universidade Federal de São Paulo - UNIFESP - campus São Paulo

Departamento de Oncologia Clínica e Experimental Rua Doutor Diogo de Faria, 824 - Vila Clementino São Paulo, SP, Brasil - CEP 04037-002

E-mail: nutricaooncologia@gmail.com

\section{Submissão}

30 de setembro de 2019

\section{Aceito para publicação}

21 de fevereiro de 2020

\begin{abstract}
RESUMO
Introdução: Em pacientes oncológicos, o risco de desnutrição é constante devido a vários fatores, como balanço energético negativo e perda de massa musculoesquelética. Os tratamentos oncológicos, frequentemente, produzem diversos efeitos colaterais, que afetam diretamente a ingestão alimentar, representando risco nutricional. Como estratégia, prescreve-se suplementos industrializados, entretanto, devido às náuseas e à disgeusia comumente encontradas nestes pacientes, a tendência é a aceitação de versões sem sabor. Objetivos: Realizar avaliação sensorial cega em preparações culinárias acrescidas de suplementos industrializados em pó sem sabor. Método: Produziu-se 3 receitas, em laboratório de Técnica Dietética da UNIFESP, um bolo de fubá, uma omelete simples e um creme de abacate. Cada receita foi repetida 4 vezes, sendo que em 3 delas foi adicionado suplemento de 3 marcas diferentes e uma sem adição. As preparações foram submetidas a um estudo experimental cego, dispostas em recipientes semelhantes, numeradas e solicitou-se aos indivíduos que degustassem os alimentos atribuindo notas de 1 a 5 para os critérios de aparência, sabor, aroma e textura. Os indivíduos serviram-se de forma aleatória, sem saber quais preparações continham o suplemento ou a qual marca pertenciam e preencheram o formulário de avaliação sensorial. Resultados: Submeteram-se ao teste sensorial 14 indivíduos. Notou-se que as preparações doces com suplemento, como o bolo de fubá e creme de abacate, foram melhor aceitas, com aprovação média de $86 \%$ e $76,3 \%$, respectivamente, enquanto a omelete obteve $51,6 \%$. Verificou-se, também, que todos os indivíduos conseguiram perceber a presença de suplemento na omelete e no creme de abacate e, no bolo de fubá, com menor frequência. Conclusão: A orientação dos fabricantes é de não submeter os suplementos a altas temperaturas, pelo risco de perda nutricional, entretanto, no relato de pacientes, notam-se que a inserção de suplemento em alimentos prontos é perceptível, a proposta seria uma alternativa de uso enriquecendo as preparações diárias.
\end{abstract}

\section{ABSTRACT}

Introduction: In oncology patients, the risk of malnutrition is constant due to several factors, such as negative energy balance and losses of musculoskeletal mass. Cancer treatments often results in several side effects that directly affect food intake, representing nutritional risk. As a strategy, industrialized supplements are prescribed, however, due to the nausea and dysgeusia commonly found in these patients, the tendency is to accept versions flavorless. A blind sensory evaluation was performed using culinary preparations increased with flavorless powdered industrial supplements. Methods: Three recipes were produced in an UNIFESP Food Technology Laboratory, a cornmeal cake, a simple omelet and an avocado cream. Each recipe was repeated 4 times, in 3, added supplement of 3 different brands and one without addition. The preparations were arranged in similar containers identified by numbers and assessors were asked to taste the food by giving grades 1 through 5 for appearance, taste, aroma and texture. The assessors served themselves randomly, not knowing which preparations contained the supplement or which brand they belonged to and filled out the sensory evaluation form. Results: Fourteen individuals were submitted to the sensorial test. It was noted that sweet supplemented preparations, such as cornmeal cake and avocado cream, were better accepted, with average approval of $86 \%$ and $76.3 \%$, respectively, while omelet obtained $51.6 \%$. It was also found that all individuals were able to notice the presence of supplement in the omelet and avocado cream, and less frequently in cornmeal cake. Conclusion: The brand's orientation is not to submit supplements to high temperatures, because of the risk of nutritional loss, however, in patient's reports, the insertion of supplement in preparations is noticeable, the proposal would be an alternative use to enrich the daily preparations.

1. Doutoranda em Ciências da Saúde na área de Oncologia na Universidade Federal de São Paulo (UNIFESP), Mestrado em Ciências da Saúde na área de Oncologia na UNIFESP, Especialista em Nutrição Clínica - GANEP, Graduação em Nutrição na Universidade São Judas Tadeu, São Paulo, SP, Brasil.

2. Residente do Programa de Residência Multiprofissional em Oncologia da Universidade Federal de São Paulo (UNIFESP), Graduaçãa em Nutrição na Universidade Federal de Alfenas (UNIFAL), onde foi bolsista da Coordenação de Aperfeiçoamento de Pessoal de Nível Superior (CAPES), com período sanduíche na Northumbria University (2014/2015), São Paulo, SP, Brasil.

3. Residente do Programa de Residência Multiprofissional em Oncologia - UNIFESP, Graduação em Nutrição pela Universidade de São Paulo, São Paulo, SP, Brasil.

4. Residente do Programa de Residência Multiprofissional em Oncologia da Universidade Federal de São Paulo (UNIFESP), Especialista em Nutrição Esportiva pelo Centro de Estudos de Fisiologia do Exercício e Treinamento (CEFIT), Graduação em Nutrição pela Universidade de São Paulo, onde foi bolsista da Coordenação de Aperfeiçoamento de Pessoal de Nível Superior (CAPES), com período sanduíche na La Salle University (2015/2016), São Paulo, SP, Brasil.

5. Professora Doutora do Departamento de Biociências do curso de Nutrição da Universidade Federal de São Paulo (UNIFESP) - campus Santos, Santos, SP, Brasil. 


\section{INTRODUÇÃO}

Os suplementos orais têm sido utilizados em pacientes com os mais diferentes tipos de patologias, sendo internados ou em atendimento ambulatorial, apresentando risco nutricional ou desnutridos, como estratégia para melhorar seu prognóstico.

Em pacientes oncológicos, especificamente, o risco de desnutrição é constante, devido a vários fatores, como balanço energético negativo e perda de massa musculoesquelética, que podem ocorrer por meio da combinação entre a redução da ingestão alimentar, aumento da taxa metabólica basal, resistência à insulina, lipólise, proteólise, efeitos colaterais relacionados ao tratamento, inflamação sistêmica e fatores catabólicos que podem ser provenientes do indivíduo ou derivados do tumor ${ }^{1}$.

A quimioterapia e/ou a radioterapia são tratamentos frequentemente propostos para doenças oncológicas, e podem provocar uma diversidade de efeitos colaterais, como náuseas, êmese, anorexia, disgeusia, xerostomia, diarreia, e tantos outros que prejudicam diretamente a ingestão alimentar do doente, elevando seu risco nutricional consideravelmente ${ }^{2}$.

Há no mercado diversos suplementos industrializados em forma líquida ou em pó de diversos sabores, entretanto, devido aos efeitos colaterais relatados acima, o paciente oncológico pode apresentar dificuldade na aceitação dos mesmos.

Em nossa prática clínica é muito comum recebermos a queixa de pacientes em relação ao uso de suplementos. São situações variadas, desde a dificuldade de aquisição, sabor, volume de consumo, entre outras, que podem reduzir a adesão e elevar o risco nutricional.

Neste sentido, o uso de suplementos em pó sem sabor tem se mostrado promissor, entretanto, percebe-se que muitos pacientes rejeitam acrescentar o produto no alimento pronto, conforme indicado pelos fabricantes.

Existem, atualmente, três opções de suplementos em pó sem sabor, são eles: Nutren Sênior ${ }^{\circledR}$ da marca Nestlé ${ }^{\circledR}$, Nutridrink Max ${ }^{\circledR}$ da marca Danone ${ }^{\circledR}$ e Inmax ${ }^{\circledR}$ da marca Prodiet ${ }^{\circledR}$. As empresas orientam a adicionar estes produtos em preparações salgadas ou doces, após o preparo, e, pela perda de nutrientes que pode ocorrer na cocção, não devem ser submetidos a altas temperaturas.

Entretanto, não obtivemos, com nenhuma destas empresas, a quantidade de nutrientes perdidos nestes suplementos quando submetidos ao aquecimento. Buscando uma nova opção de indicação, pensamos em utilizar os suplementos diretamente em preparações culinárias, submetendo-os a condições diferentes de preparo e aquecimento ou resfriamento, realizando avaliação sensorial, por meio de teste cego, verificando aparência, sabor, aroma e textura destes alimentos, comparando-os com preparação semelhante, entretanto, sem a inclusão do suplemento.

\section{MÉTODO}

Foram selecionados três tipos diferentes de preparações culinárias para o teste com os suplementos sem sabor, com base em três preceitos: deveriam ser de custo baixo, de simples preparo, para que o paciente e/ou cuidador conseguissem reproduzi-las e ter tipos diferentes de cocção ou preparo, para isto, selecionamos uma receita grelhada, uma assada e uma gelada, esta última não foi submetida a aquecimento.

As preparações foram elaboradas no Laboratório de Técnica Dietética da Universidade Federal de São Paulo UNIFESP, campus Santos e as selecionadas foram: bolo de fubá (Receita 1), omelete simples (Receita 2) e creme de abacate (Receita 3), em todas estas receitas consta a inserção

\section{RECEITA 1}

Bolo de Fubá

\begin{tabular}{lcc}
\hline Ingrediente & Peso $(\mathrm{g} / \mathrm{ml})$ & Medida caseira \\
\hline Farinha de trigo & $117 \mathrm{~g}$ & 1 xícara de chá \\
Leite integral & $106 \mathrm{ml}$ & $1 / 2$ xícara de chá \\
Ovo & $108 \mathrm{~g}$ & 2 unidades \\
Fubá & $93 \mathrm{~g}$ & $3 / 4$ de xícara de chá \\
Margarina & $15 \mathrm{~g}$ & 1 colher de sopa \\
Açúcar & $113 \mathrm{~g}$ & $3 / 4$ de xícara de chá \\
Fermento em pó & $6,5 \mathrm{~g}$ & $1 / 2$ colher de sopa \\
Suplemento em pó & $27,5 \mathrm{~g}$ & 3 colheres de sopa
\end{tabular}

\section{Modo de preparo:}

Bata as claras em neve e separe. Inicialmente, bata na batedeira o óleo, a margarina, o açúcar e as gemas. Acrescente na sequência a farinha de trigo, o leite, o fubá, o suplemento, o fermento em pó e, por último, mexendo delicadamente, as claras em neve. Deve ser acondicionado em forma pequena untada e enfarinhada e assada em forno médio $\left(180^{\circ} \mathrm{C}\right)$ até o completo cozimento interno.

\section{RECEITA 2}

Omelete

\begin{tabular}{lcc}
\hline Ingrediente & Peso $(\mathrm{g} / \mathrm{ml})$ & Medida caseira \\
\hline Ovo & $54 \mathrm{~g}$ & 1 unidade \\
Sal & $880 \mathrm{mg}$ & 1 pitada \\
Suplemento em pó & $27,5 \mathrm{~g}$ & 3 colheres de sopa
\end{tabular}

\section{Modo de preparo:}

Misturar todos os ingredientes em um recipiente e colocar em frigideira com um fio de óleo, suficiente para não grudar e aquecer dos dois lados até o completo cozimento. 
RECEITA 3

Creme de Abacate

\begin{tabular}{lcc}
\hline Ingrediente & Peso $(\mathbf{g} / \mathrm{ml})$ & Medida caseira \\
\hline Leite condensado & $395 \mathrm{~g}$ & 1 lata \\
Creme de leite & $395 \mathrm{~g}$ & 1 lata \\
Leite integral & $395 \mathrm{ml}$ & 1 medida da lata \\
Abacate & $250 \mathrm{~g}$ & 1 unidade pequena \\
Suplemento em pó & $27,5 \mathrm{~g}$ & 3 colheres de sopa
\end{tabular}

\section{Modo de preparo:}

Higienize corretamente o abacate, descasque, pique e reserve. Coloque no liquidificador o leite integral, o creme de leite, o leite condensado, o suplemento e o abacate picado. Bata bem até formar um creme homogêneo. Coloque no congelador ou refrigerador para gelar.

do suplemento, na receita controle, todos os ingredientes e tempo de preparo foram idênticos, apenas não foi incluído o suplemento.

As receitas foram produzidas quatro vezes cada uma, repetindo exatamente o modo de preparo, peso dos ingredientes e tempo de cocção ou refrigeração, para que em três delas, fossem acrescidos, individualmente 27,5 gramas de suplemento em pó sem sabor, o que representa aproximadamente metade da indicação diária de consumo, das três marcas disponíveis no mercado, a saber: Nutren Sênior ${ }^{\circledR}$ da marca Nestlé ${ }^{\circledR}$, Nutridrink Max ${ }^{\circledR}$ da marca Danone ${ }^{\circledR}$ e Immax ${ }^{\circledR}$ da marca Prodiet ${ }^{\circledR}$; além disto, fizemos uma preparação idêntica, entretanto sem a inclusão do suplemento, como comparação.

Inicialmente, numeramos, aleatoriamente, os suplementos de 1 a 3, não seguindo a ordem supracitada, e o número 4 foi selecionado para ser o preparo sem a inclusão do suplemento (controle).

\section{RESULTADOS}

As preparações foram padronizadas em todos os aspectos, o bolo de fubá foi assado em 16 minutos, em forno combinado à temperatura de $180^{\circ} \mathrm{C}$, e em seu interior, a temperatura média foi de $91,2^{\circ} \mathrm{C}$, apresentando fácil dilvição dos três suplementos no preparo das massas, o peso final foi de 609 gramas.

$\bigcirc$ creme de abacate foi liquidificado por 4 minutos e refrigerado por 2 horas, tendo fácil dissolução dos 3 produtos, o volume final foi de 1367,5 ml.

Em relação à omelete, a homogeneização dos 3 suplementos foi difícil, apresentando formação de grumos, necessitando de colheres para auxiliar no processo, entretanto, foram levados ao fogo somente após a dissolução total, o tempo em frigideira foi de 4 minutos, atingindo uma temperatura interna média de $62,2^{\circ} \mathrm{C}$, tendo o peso final em torno de $65 \mathrm{~g}$.

Nota-se que houve acréscimo de valor calórico total e todos os macronutrientes em todos os preparos, comparados aos alimentos controle, conforme demonstra a Tabela 1.

Não houve diferença estatisticamente significante na comparação entre os valores encontrados entre as preparações com a inserção de suplemento.

Quanto à comparação entre os valores calóricos totais (VCT) entre os alimentos com e sem suplemento, verificou-se maior diferença em relação à omelete e menor diferença em relação ao creme de abacate ou ao bolo.

Nota-se que, nas três preparações, observando-se a quantidade de lipídeos, não há um grande acréscimo comparando-se ao alimento controle, nos três suplementos testados; em relação ao carboidrato, é possível notar grande diferença, na omelete, já que o ovo sozinho não apresenta

Tabela 1 - Composição nutricional (VCT e macronutrientes) de uma porção* dos alimentos preparados com a inserção de suplementos em pó sem sabor, em comparação aos alimentos controle.

\begin{tabular}{|c|c|c|c|c|}
\hline Preparação & VCT (kcal) & Proteínas (g) & Carboidratos (g) & \\
\hline Bolo de fubá $(1)^{\star \star}$ & 186,94 & 4,26 & 27,98 & 7,91 \\
\hline Bolo de fubá (2) & 186,61 & 4,29 & 29,71 & 7,84 \\
\hline Bolo de fubá (4) & 176,88 & 2,96 & 24,36 & 7,71 \\
\hline Omelete (1) & 294,30 & 29,51 & 43,07 & 14,46 \\
\hline Omelete (2) & 290,45 & 29,90 & 63,00 & 13,74 \\
\hline Omelete (4) & 178,25 & 14,47 & 1,29 & 12,2 \\
\hline Creme de abacate (1) & 189,45 & 4,80 & 22,51 & 10,40 \\
\hline Creme de abacate (2) & 189,17 & 4,83 & 23,97 & 10,35 \\
\hline Creme de abacate (3) & 189,61 & 4,73 & 24,12 & 10,38 \\
\hline Creme de abacate (4) & 180,97 & 3,70 & 19,45 & 10,24 \\
\hline
\end{tabular}

* uma porção da omelete corresponde a 1 preparação com 1 ovo; uma porção de bolo de fubá corresponde a 52 g e 1 porção de creme de abacate corresponde a 100 ml.

**Os números 1, 2 e 3 representam as preparações com suplemento e a 4 representa a preparação controle. 
grande quantidade de carboidrato em sua composição, provindo, então, toda a medida do suplemento.

Observando-se a composição proteica, nota-se um acréscimo considerável nas porções finais com a inserção do suplemento. Observando-se o creme de abacate, uma porção sem suplemento contém 3,70 gramas e uma porção com suplemento, em média tem 4,78 gramas, ou seja, um acréscimo de 29,18\% em sua composição proteica.

Em relação ao bolo de fubá, uma porção tem, em média, 2,96 gramas de proteína, com o acréscimo do suplemento, este valor, em média, chega a 4,24 gramas, o que representa um acréscimo de $43,24 \%$ na porção final.
A omelete é a preparação onde nota-se maior ganho proteico, já que a preparação sozinha contém 14,47 gramas de proteína, e com a inclusão do suplemento, obtém-se, em média, 29,31 gramas, correspondendo a 102,55\% de ganho proteico.

As preparações, depois de prontas, foram dispostas em recipientes idênticos, como demonstrado nas Figuras 1 a 3, numerados de 1 a 4 e submeteu-se, então, a um estudo experimental cego, onde os indivíduos, voluntariamente, propuseram-se a degustar os alimentos, de forma aleatória, sem saber qual deles tinha o suplemento e qual não continha, além de também não saber qual marca estava

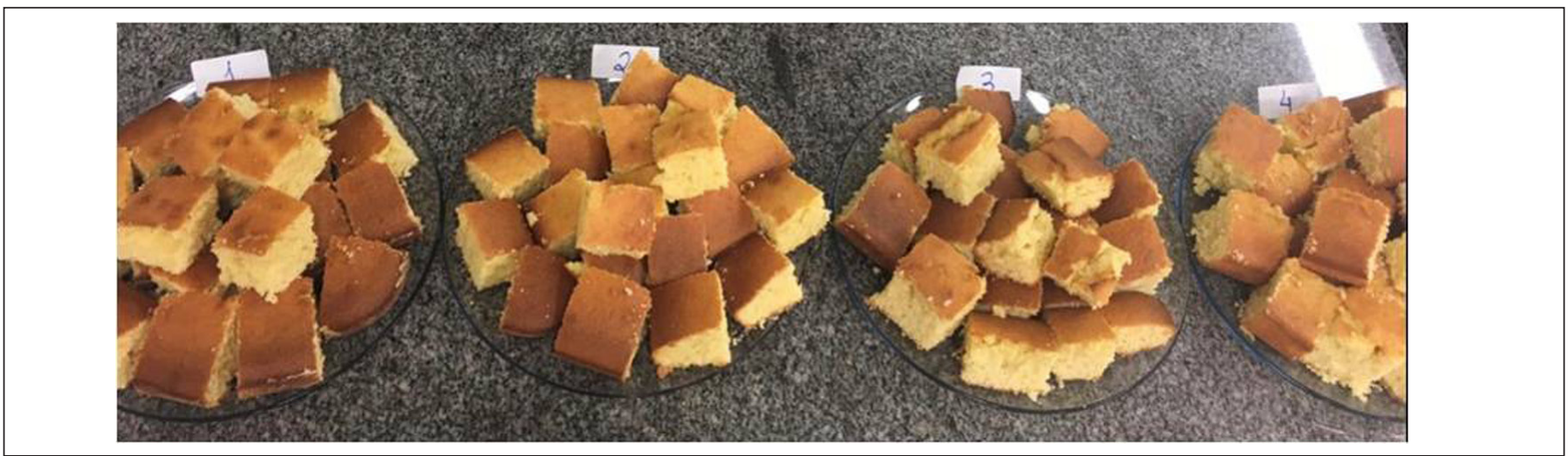

Figura 1 - Aspecto dos 4 bolos de fubá dispostos em pratos numerados de 1 a 4, onde o número 4 era a preparação sem a inclusão de suplemento.

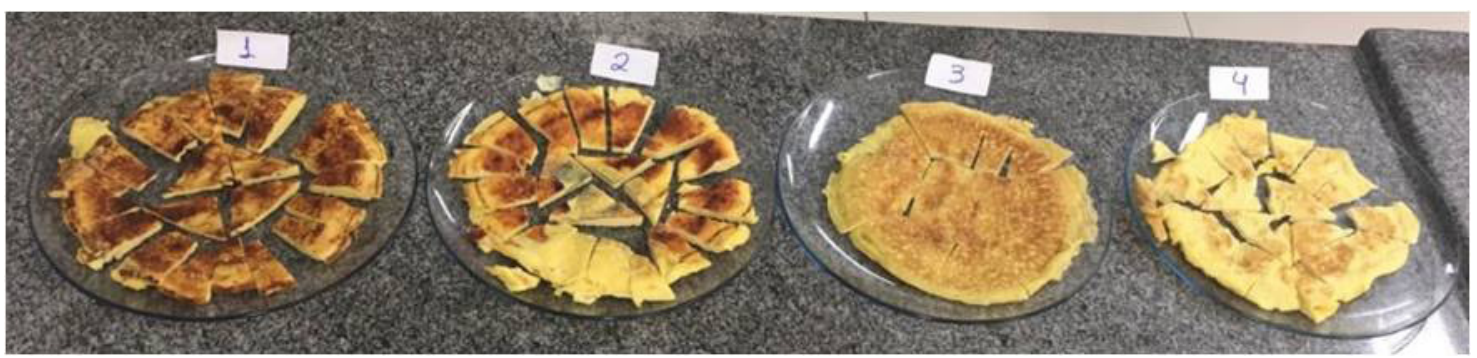

Figura 2 - Aspecto das 4 omeletes dispostas em pratos numerados de 1 a 4, onde o número 4 era a preparação sem a inclusão de suplemento.

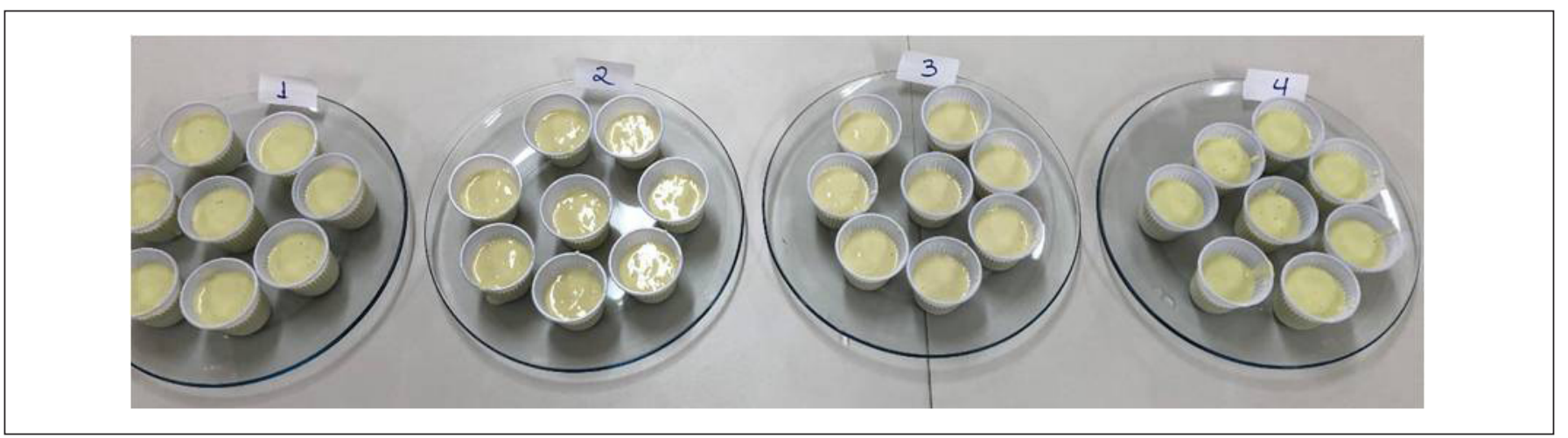

Figura 3 - Aspecto dos 4 cremes de abacate dispostos em copos plásticos em pratos numerados de 1 a 4 , onde o número 4 era a preparação sem a inclusão de suplemento. 
em cada preparo. Solicitou-se, então, que preenchessem um formulário de avaliação sensorial, atribuindo notas de 1 a 5, onde 1 significa muito ruim, 2 ruim, 3 regular, 4 bom e 5 muito bom, para 4 critérios: aparência, sabor, aroma e textura.

Submeteram-se ao teste sensorial 14 indivíduos, entre eles, professores e alunos da UNIFESP campus Santos, que estavam presentes no local e, voluntariamente, dispuseram-se a realizar a degustação.
Os resultados são apresentados nas Figuras 4 a 6 , demonstrando a média geral dos 4 critérios, aparência, sabor, aroma e textura, comparando as amostras com e sem suplemento, numeradas conforme referido anteriormente.

Notou-se que as preparações doces (bolo e creme de abacate) tiveram melhor desempenho em relação à salgada (omelete), no que diz respeito à percepção da presença ou não do suplemento, tendo, respectivamente, aprovação média de $86 \%, 76,3 \%$ e $51,6 \%$.

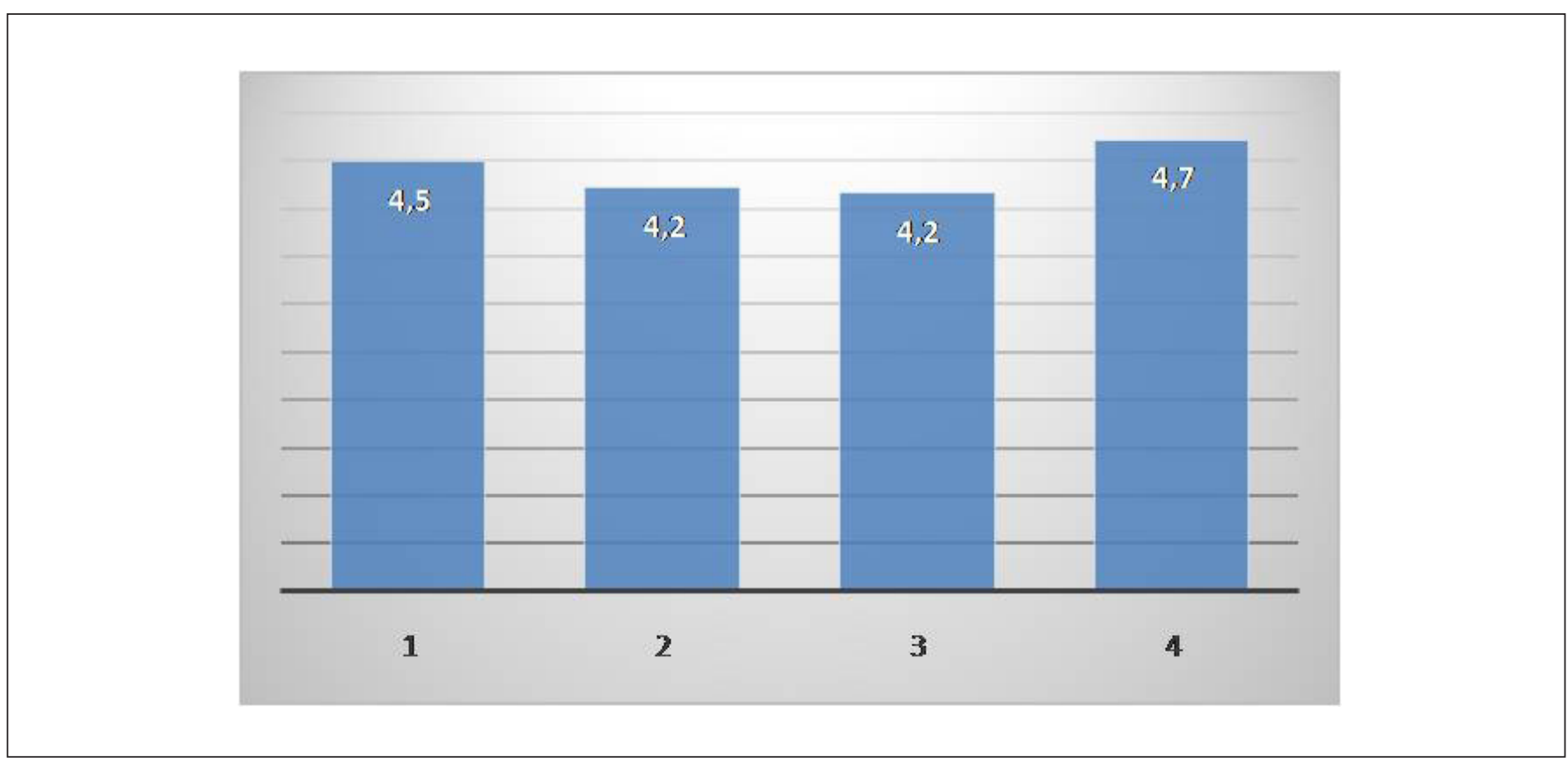

Figura 4 - Nota média comparando as 4 preparações de bolo de fubá em relação aos critérios de avaliação.

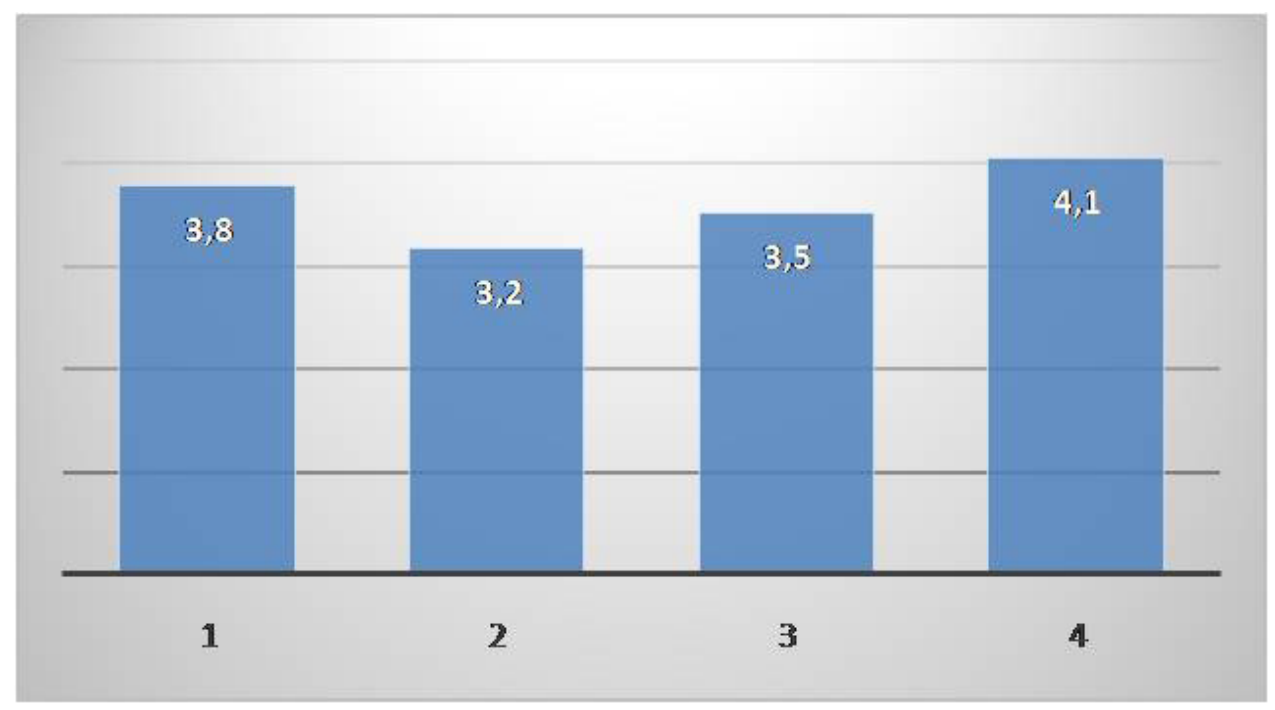




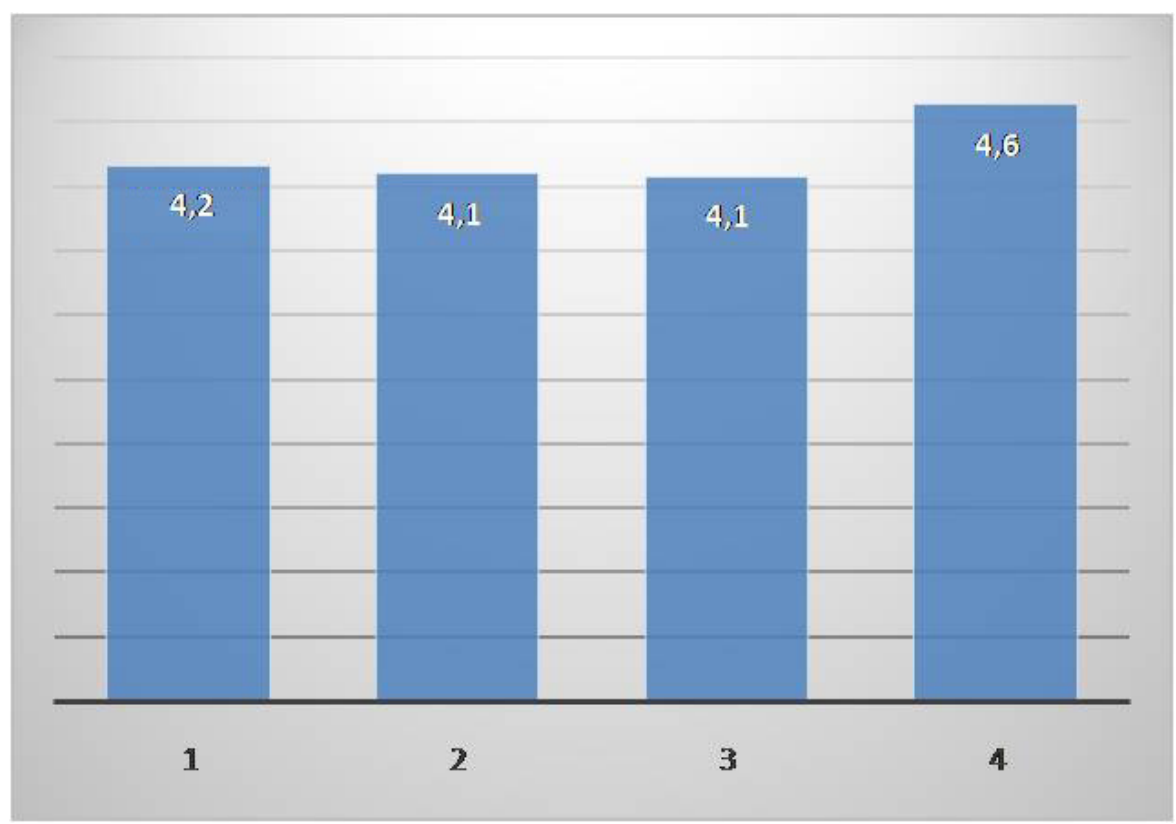

Figura 6 - Nota média comparando as 4 preparações de creme de abacate em relação aos critérios de avaliação.

\section{DISCUSSÃO}

indivíduo com câncer está sujeito a diversas alterações metabólicas, que podem agravar seu estado nutricional. Estes fatores podem estar relacionados à presença da doença em si ou ao estadiamento, ou pelos tratamentos propostos para o indivíduo, como cirurgias, quimioterapia, radioterapia, aplicados de maneira isolada ou combinados, que contribuem para a desnutrição do paciente. Com a desnutrição as possibilidades terapêuticas vão se reduzindo, uma vez que se faz necessária a redução ou suspensão de doses de quimioterapia ou de radioterapia, maior morbidade e risco em abordagens cirúrgicas, comprometendo a qualidade de vida e a sobrevida dos indivíduos ${ }^{3}$.

Sabe-se que a perda de peso, principalmente de massa magra, impacta diretamente no aumento de dias de internação, maiores riscos de infecções, de complicações cirúrgicas e de toxicidade ao tratamento quimioterápico, que podem levar à morte do paciente ${ }^{4}$.

O paciente oncológico está sujeito a diversas alterações no metabolismo de macronutrientes, como, por exemplo, nas proteínas, com o aumento no turn over, redução na síntese proteica muscular, balanço nitrogenado negativo, aumento da produção de proteínas de fase aguda, alteração do perfil dos aminoácidos no plasma 5 .

Para o paciente em tratamento quimioterápico ou radioterápico, nota-se, com certa frequência, que alguns efeitos colaterais, como náusea, êmese e anorexia, são muito comuns, muitas vezes associados a outros, como disgeusia, xerostomia, astenia, diarreia ou obstipação, que dificultam a ingestão alimentar destas pessoas, provocando diretamente a perda de peso destes indivíduos ${ }^{6}$.

Considerando-se todas estas variáveis associadas ao pior desfecho do tratamento do paciente, faz-se necessário o uso de abordagens seguras para o manejo nutricional precoce do paciente? .

As estratégias nutricionais para aumentar o consumo de alimentos são numerosas, como modificação de consistência ou textura, aumento da frequência alimentar, com redução do tamanho das porções ou, ainda, a inserção de alimentos concentrados em calorias ou proteínas, além disto, dispõe-se também dos suplementos orais industrializados, que se apresentam na forma líquida ou em pó, acrescidos de sabor ou isentos, que geralmente possuem composição de nutrientes completas para consumo, devendo ser calculadas em sua indicação diária individualmente ${ }^{8}$.

Segundo as diretrizes BRASPEN ${ }^{8}$, para o paciente oncológico, a suplementação oral está indicada para indivíduos que não conseguem atingir $70 \%$ de suas metas nutricionais utilizando somente a dieta convencional.

Guerino et al. ${ }^{9}$ avaliaram a aceitação de suplementos orais em pacientes internados, não portadores de neoplasia ou em tratamento quimioterápico, e perceberam que o consumo das versões com sabor foi bem aceita, e que dentre 
elas, a de morango obteve destaque. Nota-se, em estudo realizado com pacientes internados, que os mesmos acreditam que a ingestão do suplemento tem efeito positivo em sua saúde, e, portanto, procuram consumi-los ${ }^{10}$.

O paciente oncológico tem o mesmo intuito, ele se propõe a consumir o suplemento com a intenção de melhorar seu estado nutricional, entretanto com a cascata de efeitos colaterais a que se submetem, torna-se difícil o consumo de quaisquer alimentos, incluindo os suplementos.

Em relação aos suplementos industrializados, encontrase atualmente diversas marcas e apresentações, dentre elas as líquidas e as em pó; esta última pode ser inserida em alimentos já prontos, entretanto, encontra-se limitação nas apresentações com sabor, já que não existem versões salgadas, somente doces, e muitos deles apresentam sacarose, o que também limita o uso em indivíduos diabéticos ou que apresentam alteração de glicemia em vigência do tratamento, principalmente com o uso de medicamentos esteroides. Neste sentido, as versões sem sabor apresentam maior possibilidade de manejo no consumo, já que podem ser incluídas em preparações salgadas, e não contém sacarose em sua formulação.

Para o paciente oncológico, devido a todos os efeitos colaterais já relatados, verifica-se, pela prática clínica, que o uso das versões isentas de sabor parece ser melhor aceita, já que tendem a tolerar menos os sabores doces, permitindo ao nutricionista maior possibilidade de manejo na alimentação do mesmo.

O indivíduo, em geral, nota a presença do suplemento nas preparações que consome quando ele é incluso e, em alguns casos, refere rejeição ao alimento, pois muda a aparência, por exemplo, sucos tornam-se esbranquiçados, referem que com o sintoma de disgeusia, o alimento natural torna-se "diferente", e com a inserção do suplemento, pode ser pouco aceitável.

Pensou-se na utilização do suplemento no preparo de alimentos doces e salgados para tentar "mascarar" a presença do mesmo, buscando uma nova alternativa de consumo, e este tipo de teste não foi encontrado na literatura.

Nota-se que é positiva a presença de suplemento no alimento, pois infere acréscimo nutricional ao mesmo, como verificou-se nas preparações realizadas. Este fato demonstra a questão positiva desta prática; entretanto, os fabricantes não indicam o aquecimento de seus produtos com a justificativa de perda nutricional, mas também não se conseguiu localizar na literatura a mensuração desta perda.

Sabe-se, por exemplo, que as proteínas modificam sua estrutura física com a ação do calor, podendo passar de estrutura quaternária para primária, por exemplo, entretanto, este fato pode ser bastante positivo e desejado, iá que beneficia a digestão e absorção deste nutriente ${ }^{11}$. Comparando-se os valores calóricos totais entre as preparações com e sem suplemento, verificou-se maior diferença em relação à omelete e menor diferença em relação ao creme de abacate ou ao bolo; isto se deve ao fato de uma porção da omelete conter toda a medida de suplemento $(27,5 \mathrm{~g})$ e as outras preparações apresentarem um produto final em maior quantidade.

Seguindo esta linha de raciocínio, a composição proteica também recebeu acréscimo e, novamente, observando-se a omelete, obteve-se um valor superior ao dobro do encontrado na preparação, entretanto, a palatabilidade do produto final ficou bastante prejudicada, e esta foi a preparação que teve notas menores em relação às preparações doces. Este fato pode ter duas possibilidades, ou ser devido a maior concentração de suplemento do que as outras preparações ou, ainda, devido ao fato do aquecimento poder ter alterado o sabor do suplemento e, consequentemente, do produto final. Para responder a esta questão, mais testes devem ser realizados.

Buscou-se na literatura estudos que demonstrassem esta dificuldade de aceitação alimentar ou do suplemento que o paciente oncológico frequentemente apresenta, no entanto, não foram encontrados estudos que comprovem esta questão, e isto torna-se compreensível, já que os pacientes são indivíduos completamente diferentes, o limiar de palatabilidade é diferente, as doenças que apresentam, estadiamentos, idade, tratamento, enfim, tudo o que envolve estes indivíduos são completamente heterogêneos; estudos nesta linha devem buscar indivíduos com características o mais próximo possível uns dos outros, e mesmo assim, encontrar-se-iam resultados em um grupo específico, possivelmente não podendo ser "extrapolado" para outro grupo.

\section{CONCLUSÃO}

Segundo os fabricantes, não há recomendação para o aquecimento dos suplementos, no entanto, percebemos nos relatos de nossos pacientes que o uso diário destes produtos pode ser perceptível, monótono ou desagradável, então, nota-se que a inserção do suplemento no preparo do alimento "mascara" a presença do mesmo, podendo se tornar uma alternativa plausível de consumo. Como não houve diferença estatisticamente significante entre os produtos, decidimos não revelar qual número corresponde a qual marca, iá que para comprovarmos eventuais diferenças, sugerimos a realização de um estudo maior, com mais preparações e mais indivíduos realizando avaliação sensorial, já que isto não foi possível, por motivo de força maior, no momento do desenvolvimento deste. 


\section{REFERÊNCIAS}

1. Arends J, Bachmann P, Baracos V, Barthelemy N, Bertz H, Bozzetti F, et al. ESPEN guidelines on nutrition in cancer patients. Clin Nutr. 2017;36(1):11-48.

2. Pinho NB, coordenador. Consenso nacional de nutrição oncológica. Instituto Nacional de Câncer José Alencar Gomes da Silva. Rio de Janeiro: INCA; 2015.

3. Marín Caro MM, Laviano A, Pichard C. Nutritional intervention and quality of life in adult oncology patients. Clin Nutr. 2007;26(3):289-301.

4. Pereira A, Barrére APN. Suplementos de macro e micronutrientes. In: Baiocchi O, Sachs A, Magalhães LP, eds. Aspectos nutricionais em oncologia. Rio de Janeiro: Atheneu; 2018. p.171-8.

5. Hamilton KH. Nutritional needs of the adult oncology patient. In: Leser M, Ledesma N, Bergerson S, Trujillo E, eds. Oncology nutrition for clinical practice. Chicago: Oncology Nutrition Dietetic Practice Group; 2013. p.33-9.
6. Arends J, Baracos V, Bertz H, Bozzetti F, Calder PC, Deutz NEP, et al. ESPEN expert group recommendations for action against cancer-related malnutrition. Clin Nutr. 2017;36(5):1187-96.

7. Baldwin C, Spiro A, Ahern R, Emery PW. Oral nutritional interventions in malnourished patients with cancer: a systematic review andmeta-analysis. JNatlCancerInst. 2012;104(5):371-85.

8. Horie LM, Barrére APN, Castro MG, Liviera AMB, Carvalho AMB, Pereira A, et al. Diretriz BRASPEN de terapia nutricional no paciente com câncer. BRASPEN J. 2019;34(supl 1):2-32.

9. Guerino VL, Ferreira ACRM, Siviero L, Rabito EI. Commercial nutritional supplements sensory analysis by hospital inpatients. Nutr Clín Diet Hosp. 2018;38(2):43-8.

10. Wierzejska R, Jarosz M, Siuba M, Rambuszek M. Assessing patients' attitudes towards dietary supplements. Rocz Panstw Zakl Hig. 2014;65(4):317-23.

11. Frenhani PB, Burini RC. Mecanismos de absorção de aminoácidos e oligopeptídios. Controle e implicações na dietoterapia humana. Arq Gastroenterol. 1999;36(4):227-37.

Local de realização do estudo: Universidade Federal de São Paulo - campus São Paulo, São Paulo, SP, Brasil.

Conflito de interesse: Os autores declaram não haver.

Trabalho apresentado no $23^{\circ}$ Congresso Brasileiro de Nutrição Parenteral e Enteral, realizado em Foz do Iguaçu, PR, Brasil, em 21 de outubro de 2019. 